\title{
Proxemics Revisited: Similar Effects of Arms Length on Men's and Women's Personal Distances
}

\author{
Nicola Bruno ${ }^{1, *}$, Michela Muzzolini ${ }^{2}$ \\ ${ }^{1}$ Dipartimento di Neuroscienze, Unità di Psicologia, Università di Parma, Borgo Carissimi 10, 43100 Parma, Italy \\ ${ }^{2}$ Dipartimento di Scienze della Vita, Unità di Psicologia "Gaetano Kaniza", via Weiss 21, 34178 Trieste, Italy \\ * Corresponding Author: nicola.bruno@unipr.it
}

Copyright $(2013$ Horizon Research Publishing All rights reserved.

\begin{abstract}
Handbooks of human communication describe personal distance as a protective bubble surrounding our body and stress the role of sex (along with other cultural and situational factors) in stretching and shrinking it. Using data from a simulated dyadic interaction procedure, we show that putative effects of sex may be explained by effects of one's arm length in same-sex and different-sex interactions. This suggests that biological factors related to the body schema provide the crucial constraints on the use of personal space for communication.
\end{abstract}

Keywords Proxemics, Personal distance, Sex, Body Schema

\section{Introduction}

Distance plays an obvious role in social communication. Most of us have experienced discomfort or annoyance when an interlocutor comes "too close", and have learned to modulate interpersonal distance to signify intimacy, or threat, in nonverbal communication. Hall coined the term "proxemics" for the study of the role of distance in social interactions [1]. He argued that human interactions are constrained by four types of proxemic spaces: intimate (from 0 to about $0.5 \mathrm{~m}$ ), personal (0.5 to $1.2 \mathrm{~m}$ ), social (1.2 to $3 \mathrm{~m}$ ), and public (greater than $3 \mathrm{~m}$ ). Borrowing the notion from earlier studies on animal behavior [2-3], Hall further suggested that personal space defines a sort of protective bubble around our bodies. Letting a conspecific enter the bubble signifies trust and willingness to let the other touch us, a message with various social and emotional implications. Thus, if a stranger gets too close, we are upset [4-10].

Proxemic theory has generated an impressive amount of research [11], and a substantial portion of this literature has investigated personal space and the factors that modulate the proxemic bubbles surrounding our bodies [12-14]. In this paper, we will focus on one of these factors, the sex of the individuals involved in the social interaction. It has often been reported that cultural and social norms associated with sex roles are crucial in modulating personal distance. Male-male pairs reportedly tend to interact at greater personal distances, presumably due to negative views of men who have close relationships with other men; whereas female-female pairs tend to stay closer, thus expressing more intimacy and affection; and male-female pairs tend to stay even closer, as this interaction corresponds to "the ultimate in intimate relations" [15]. Assesments of personal distance as a function of several personal and social characteristics reportedly reveal, among other effects, that shorter distances are preferred by "warm" individuals and by females [16].

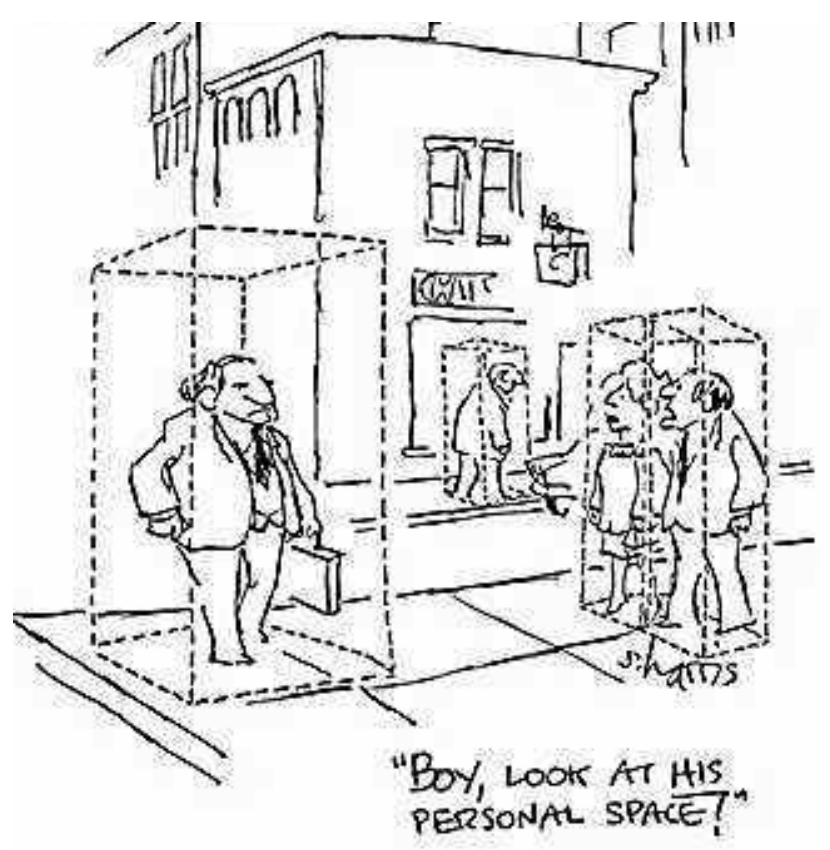

Figure 1. Distance plays a role in social communication. Reproduced from www.CartoonStock.com.

Findigs such as these have been discussed in early [17] and recent studies [18] and feature conspicuously in handbooks of human communication [19]. Hall [1] also suggested that personal space may also be related to one's reaching ability, and that in general sensory and motor constraints may have a role in proxemics. However, Hall's 
work remained ambiguous concerning the relative contributions of basic biological factors in defining proxemic distances [20-21] and later investigations have largely neglected the issue. This is surprising, as the widely reported sex difference could in principle be explained by the notion that personal space is related to our body's possibilities for action.

The concept of a "body schema" has long been used in the neuroscience literature to refer to the set of internal representations concerning one's body parts and movements [22-24]. Numerous studies have suggested that body schema-related factors, such as possibilities for grasping and reaching, can affect the representation of peripersonal space [25-26]. In particular, it appears that the transition between peripersonal ("near") space and more distant space is specifically related to arm length: individuals with longer arms show a more gradual shift in spatial biases with increasing distance than those with shorter arms [27]. Now assume that body schemas encompassing wider spans (i.e, of taller people having longer arms) imply larger proxemic personal bubbles. Then one would expect to observe that most women stand closer than most men; but not for reasons related to sex roles. One would tend to make such observation simply because men, on the average, have longer arms. To our knowledge, no published investigation has sought to assess the effect of body size on proxemic personal space.

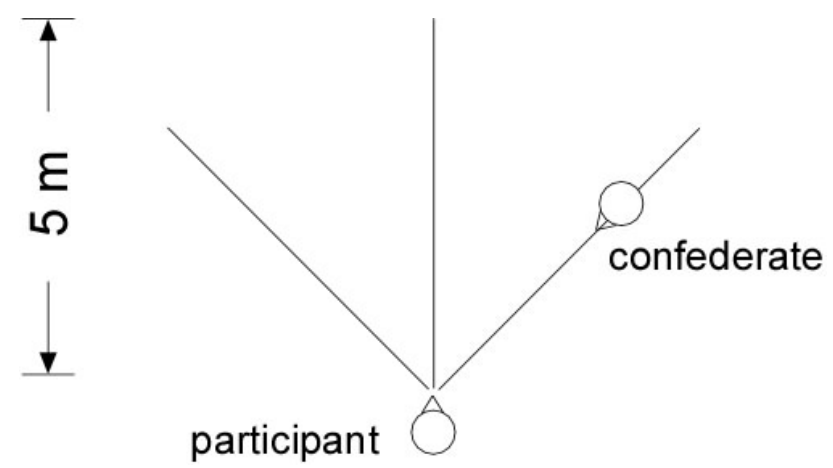

Figure 2. Spatial layout for the simulated dyadic interactions in the current study. Predetermined paths were taped on the ground. Participants mantained fixation straight ahead.

In the present paper, we seek to determine to what extent sex differences in proxemic distances during dyadic interactions could be accounted for by differences in arm length. Our approach is based on a single assumption, namely, that most of us experience discomfort when personal space is invaded, and can report it. Thus, we set up a standardized procedure whereby a confederate enters an experimental participant's personal space, and the job of the participant is to simply to report when he or she feels uneasy. Variations of this "stop distance" paradigm have often been used to obtain measures related to personal space [14, 28]. We set up our own version to include different sex pairs as well as some variation of situational factors while controlling familiarity, and collected estimates of stop distances together with measures of the participants' arm lengths. If proxemic theory as generally presented in handbooks of human communication is correct, proxemic distances should change as a function of sex quite independently of people's size. Alternatively, if arm length is crucial, proxemic distances should be modulated by this factor independently of the participant's sex.

This is indeed what we found, with an evolutionary twist. In same-sex interactions, personal distance was modulated by arms length, independently of sex. The longer the arms of the participant, the larger the distance in both men and women. In different-sex interactions, however, personal distance was modulated by the similarity of participant and confederate arm length. Both men and women participants kept at closer distances when their arms were more similar to their confederate's arms, and at longer distances when they were more different. We suggest that these effects may reflect biologically-constrained tendencies to modulate space according to two adaptive principles: i) in members of the same sex, arm length modulates personal space in accordo with possibilities for action; ii) in members of different sexes, with behavior related to accepting, or rejecting, intimacy.

\section{Materials and Methods}

\subsection{Participants and Design}

We tested a total of 124 University of Trieste psychology students, equally split between female and males, who participated for course credit. None were foreign students. Their age range was $19-27$ years with an average of 23.4 years. All were tested individually. In addition, two confederates participated in the study, one male and one female. Confederates were both 24 years old and were selected to minimize the probability that some of the participants knew them or had seen them before. In addition, the height of confederates was approximately equal to the average height of female and male participants, respectively. Participants and confederates were paired into four, equally sized groups: female-female, male-male, female with male confederate, male with female confederate.

\subsection{Materials and Procedure}

Tests were performed in a large open courtyard beneath a University of Trieste building or in a relatively large room (approximately 40 square meters) with no furniture. In both cases, three white adhesive strips, 5 meters long, were laid out on the ground to mark predetermined walking paths (see Figure 1). Participants stood at the meeting point of the strips and had to face straight ahead throughout the experiment. Confederates fixated the participants on the face and walked either forwards (personal space intrusion) or backwards (extrusion) following the predetermined paths. Instructions stressed that confederates should walk naturally at a steady 
pace and not too fast. Depending on walking direction, participants indicated the distance at which they started to feel discomfort or ceased to feel it. This report was provided by simply uttering the word "stop". As soon as the confederate stopped, the experimenter measured the distance from the centers of the confederate's and the participant's facing midsections with a semi-rigid windup meter tape, taking care to keep the meter horizontal.

Each participant contributed a total of 12 measures of personal distance, resulting from the factorial combination of 2 repetitions, 2 walking directions, and 3 predetermined paths. The order of presentation of each trial was completely randomized within each participant. Before beginning measurements, the experimenter demonstrated the task with the confederate and explained that the study aimed at measuring distances whereby participants might feel discomfort. After the experiment, the experimenter recorded the participant's heights and arm length from shoulder to wrist. Finally, she asked two additional questions: "Overall, did you feel discomfort when the confederate was close: definitely, some, or not at all?" and "On a 1- 7 scale, how familiar would you say the confederate was to you (1, complete stranger; 7, very familiar)?" and recorded the answers. Finally, participants that so desidered were briefed to the aims of the study and given a certificate for partial credit.

\section{Results}

\subsection{Data Validation}

To check for transcription errors or other potential problems in the recorded distances, we examined the histogram portraying the distribution of this variable. This distribution proved asymmetrical with a slight right tail (skewness $=1.27$ ) and a somewhat narrow central hump (kurtosis = 3.3). The five-number summary of the distribution was as follows: minimum $=6 \mathrm{~cm}$; first quartile $=$ $36 \mathrm{~cm}$; median $=60 \mathrm{~cm}$; third quartile $=87 \mathrm{~cm}$; maximum $=$ $310 \mathrm{~cm}$. Despite this wide range in results, no datapoint appeared as an obvious outlier on the histogram. For this reason, none was excluded from the analysis at this stage (but see section on Individual differences below). To check for errors in the recorded biometric variables, we plotted arm length as a function of height and inspected the bivariate distribution. As expected, these two variables were highly correlated, $\mathrm{r}=0.87$, and approximately normally distributed. Inspecting the scatterplot revealed one woman participant that was $151 \mathrm{~cm}$ tall but reportedly had arms that were $62 \mathrm{~cm}$ long (a typical arm length for a man $180 \mathrm{~cm}$ tall). Given that this was obviously a transcription error, we dropped this datapoint and run an additional female participant to substitute it.

\subsection{Familiarity}

Although confederates were chosen to minimize the probability that participants had seen them before, this possibility cannot be ruled out a priori. To evaluate the possibility of familiarity relations between participants and confederates, we inspected the frequency distribution of the familiarity scores as recorded from the relevant final question. For essentially all participants, these scores were either 1 (122 cases) or 2 (11), indicating no familiarity with the confederates. The one remaining case scored a 3 , indicating some weak degree of familiarity. We also noted that the average distance in the 111 cases who declared the candidates total strangers (score $=1$ ) turned out to be $66 \mathrm{~cm}$ $\pm 1 \mathrm{~cm}$, whereas the average in the other 12 cases (scores $=2$, 3) was slighly less than $62 \mathrm{~cm} \pm 3 \mathrm{~cm}$. (Here and elsewhere, averages are reported along with their standard errors.)

Although this difference is hard to evaluate given the lack of precision in the second estimate, it is nonetheless suggestive that even a very small difference as that characterizing the change from defining someone a total stranger to declaring some very weak degree of familiarity appears to bring down the average personal distance somewhat. At least qualitatively, therefore, this outcome is in keeping with previous reports of familiarity effects on personal distance [29]. Given the distribution of the scores and the variability in the distances, however, we concluded that in our study familiarity with the confederates was essentially constant and therefore excluded it from further analysis.

Table 1. Distribution of answers to the question: "Overall, did you feel discomfort when the confederate was close: definitely, some, or not at all?" and associated average distances at which participants stopped the confederates.

\begin{tabular}{|c|c|c|c|}
\hline answer & cases & average $(\mathrm{cm})$ & SE $(\mathrm{cm})$ \\
\hline definitely & 95 & 77.9 & 1 \\
\hline some & 11 & 34.18 & 1.3 \\
\hline not at all & 18 & 20 & 0.5 \\
\hline
\end{tabular}

\subsection{Individual Differences in Discomfort}

Although feelings of discomfort or embarassment after invasion of our personal space are commonly reported, it is to be expected that a degree of individual variability exists in the sensitivity to such phenomena. To evaluate the importance of such potential variability and its impact on personal distances in our procedure, we examined the frequency distribution of the answers to the relevant final question. As shown in Table 1, the large majority of participants reported discomfort, but a substantial minory did not or did to a reduced degree. These self reports turned out very predictive of personal distances as recorded in our simulated procedure: individuals who did not experience discomfort or did to a lesser degree let confederates come much closer than individuals that did. Given that participants were naïve to the hypotheses and had no information about our expectations about "correct" distances, these results 
provide concurrent validation to the procedure used here to assess distance causing discomfort. Whatever the reason for these individual differences, the crucial point here is that only individuals actually experiencing the phenomenon of interest can provide useful information to test our hypotheses. For this reason, all additional analyses were perfomed on these individuals only.

\subsection{Situational Effects}

Measures of personal distance were taken under different conditions. Specifically, about one third of participants was tested in an open courtyard, the remaining two thirds in a closed room. In addition, each participant reported distances along his or her line of sight, with the conferedate in the central visual field, or along paths that were off to the right or left by 45 degrees, placing the confederate in the peripheral visual field. Finally, half of the reported distances pertained to trials whereby the confederate approached the participant (intrusion trials), whereas the other half on trials whereby he or she receded walking backwards (extrusion). All these variations may entail differences in reported distances that are not of interest for the present purposes, but that may need to be controlled statistically. To evaluate this, we computed average distances as a function of the relevant levels of these three variables. As stated in the previous section, here and in all remaining analyses only participants reporting discomfort were included. The results indicated that average personal distances were generally smaller in the outdoor setting (72.1 $\mathrm{cm} \pm 1.9 \mathrm{~cm})$ than in the closed room $(79.8 \mathrm{~cm} \pm 1.3 \mathrm{~cm})$ and in intrusion $(70.4 \mathrm{~cm} \pm 1.6 \mathrm{~cm})$ than in extrusion trials $(85.4$ $\mathrm{cm} \pm 1.3 \mathrm{~cm}$ ). In addition, average distances were larger when the confederate was in the central visual field $(85.3 \mathrm{~cm}$ $\pm 2.2 \mathrm{~cm})$ than when he or she was in the periphery $(74 \mathrm{~cm} \pm$ $1.6 \mathrm{~cm}$, both on the left and on the right path). Whatever their casuses, these results indicate that variations in situational factors do indeed modulate reported personal distances, and will need to be taken into account in statistical evaluations of the variables that are the object of the current study.

\subsection{Effect of Participant-confederate Pair}

According to proxemic theory, personal distance in dyadic interactions should vary as a function of the sex of the involved individuals. In particular, within the proxemics framework man-man pairs are expected to prefer larger distances than woman-woman pairs, and mixed pairs to prefer the smallest distance of all (Evans \& Howard, 1973). To test this prediction, we computed the average personal distances in the four types of interactions occurring in our procedure: both participant and confederate male, both female, male participant with female confederate, and female participant with male confederate. Table 2 presents the results: as expected by proxemic theory, we observed larger distances in male-male than female-female pairs, and the smallest distances in pairs whereby the participant was male and the confederate female. In contrast with proxemic theory, however, in the other kind of mixed pair the average distance was larger than that for female-female pairs. We will not discuss further these results here as we believe that the crucial variable is in fact not the sex of either individual, but arm length (see following section).

Table 2. Average distances at which participants stopped the confederates as a function of their respective sexes.

\begin{tabular}{|c|c|c|c|}
\hline pair & cases & average $(\mathrm{cm})$ & SE $(\mathrm{cm})$ \\
\hline female-female & 25 & 70.7 & 1.5 \\
\hline male-female & 20 & 67.6 & 2.2 \\
\hline female-male & 23 & 79.5 & 2.3 \\
\hline male-male & 27 & 90.7 & 0.5 \\
\hline
\end{tabular}

\subsection{Effect of Arm Length}

As argued in the introduction, one reason for differences in chosen personal distances between men and women may be that men have, on the average, longer arms than women. To evaluate this possibility, we plotted results from all trials against the length of the participant's arms, after color-coding the datapoints according to the type of participant-confederate pair (see Figure 2). This plot revealed a large variability in chosen distances, as one would expect given the differences due to the situational factors examined above. Importantly for our present purposes, however, the plot also indicated that chosen distances were modulated by the length of the participant's arm.

Consider same-sex interactions first. Strikingly, in both same-sex groups the scaling of distance with arm length (as measured by the slope of least-squares regression line) amounts to an increase of about 3-4 cm in personal distance for each additional $\mathrm{cm}$ in arm length. When the male and female groups are placed on the same graph, as we have done in Figure 3, the corresponding regression fits are almost perfectly collinear, and they would largely coincide with the single regression line fitted to the two groups as a whole, had we placed it on the graph. These results indicate that, in same-sex pairs, variations in stop distances can be ascribed to differences in arm length between the participants rather than their sex: the average difference between distances in the two groups is simply due to the fact that male generally have longer arms, such that the corresponding data are displaced to the right of the graph.

Now consider different-sex interactions. In this case, the fitted lines are clearly not collinear. In fact, they seem to reveal opposite effects of arm length: when the participant was female and the confederate was male, distances decrease with the female participant's arm length, whereas they increase in the other group. However, another feature of our data suggests that arm length remains crucially involved in modulating the effect. Recall that confederates were chosen to have approximately average heights (and therefore arm lengths) for their corresponding groups. This entails that, in different-sex pairs, the male confederate was always taller 
(and had longer arms) than any female participant, whereas the female confederate was always shorter (and had shorter arms) than any male participant (see also arrows on top of Figure 3). This feature of the data suggests that, in different-sex pairs, distances did not increase as a function of the participant's arm length, but of the (unsigned) difference between the arm lengths of the participant and the confederate. Shorter female participants were more different than the male confederate and, other things being equal, kept him at a greater distance than taller female participants. Conversely, taller male participants were more different than the female confederate, and kepth her at a greater distance than shorter male participants.

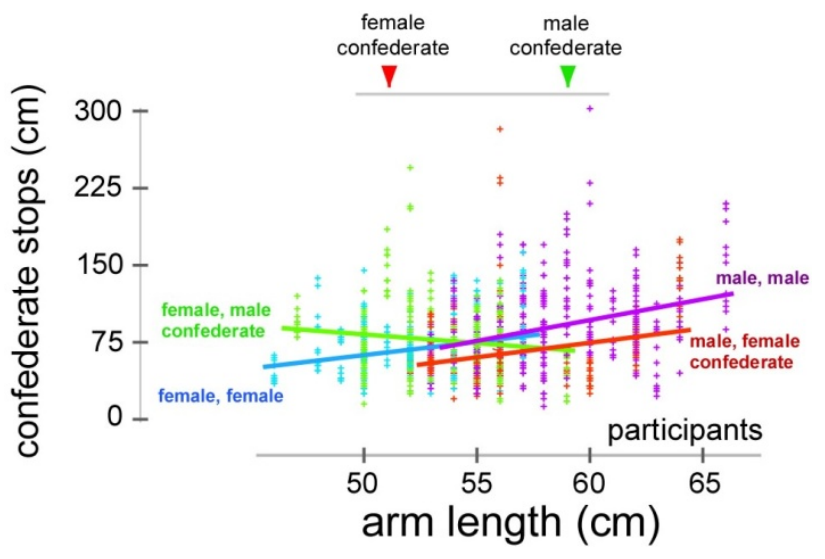

Figure 3. Chosen personal distance, as operationalized by distances whereby participants asked confederates to stop, as a function of participant arm length, in four different types of dyadic interactions. Arrows on top of the panel indicate arm lengths for the female and male confederate.

\subsection{Modelling Factors That Affect Distance}

The preliminary analyses performed in the preceding sections suggests that factors affecting distance in personal space should be described by taking into account a variety of predictors, including individual differences and various categorical factors such as the place of testing (indoors or outdoors), the direction of the confederate movement (intrusion or extrusion), and the confederate position relative to the participant line of sight (central visual field, or peripheral to the left or right). In addition, and most importantly, it suggests that the variability in chosen distances is modulated by arm length in different ways for the same-sex and different-sex pairs. Given this structure in the data, to subject the present data to inferential analysis we fitted two separate linear models (ANCOVA) to the same-sex and different-sex data, including two different quantitative covariates: in the same-sex data, we entered into the analysis the length of the participant's arm, whereas in the different-sex data we entered the absolute difference between the participant and the confederate arm lengths. Quantitative variables related to arm length were modelled as random effects varying between participants. Place of testing was modelled as a fixed effect also varying between participants. The other two categorical predictors, confederate direction and position, were modelled as fixed effect varying within participants. To provide appropriate denominators for testing within-factor effects, we also included in the model the appropriate two-way interactions with the participant factors. Finally, to compare the roles of the arm length-related and sex factors, we also entered into each analysis the participant's sex as a fourth categorical variable.

\subsubsection{Same-sex Model}

Overall, the model accounted for about $70 \%$ of the total variance. The following factors were statistically significant: arm length, $\mathrm{F}(1,416)=13.3, \mathrm{p} \leq 0.0003$, starting position, $\mathrm{F}$ $(2,102)=17.0, p \leq 0.0001$, direction of approach, $F(2,51)=$ $36.9, \mathrm{p} \leq 0.0001$, and place of testing, $\mathrm{F}(1,416)=5.9, \mathrm{p} \leq$ 0.02 . Participant sex failed to reach significance, $F(1,416)=$ $2.8, \mathrm{p}>0.09$.

\subsubsection{Different-sex Model}

Overall, this second model accounted for about $86 \%$ of the total variance, this time with all factors statistically significant: difference between participant's and confederate's arm lengths, $\mathrm{F}(1,344)=93.2, \mathrm{p} \leq 0.0001$, starting position, $\mathrm{F}(2,102)=17.0, \mathrm{p} \leq 0.0001$, direction of approach, $\mathrm{F}(2,51)=36.9, \mathrm{p} \leq 0.0001$, place of testing, $\mathrm{F}(1$, $416)=5.9, \mathrm{p} \leq 0.02$, and sex, $\mathrm{F}(1,344)=70, \mathrm{p} \leq 0.0001$.

\section{Discussion}

At least in simulated dyadic interactions such as those studied here, there is little support for the claim that sex, per $\mathrm{se}$, is a factor in modulating personal distance as claimed by standard proxemics textbooks. The present pattern of results suggests instead that an important difference exists in the use of personal sapce between pairs of individuals of the same sex and of different sexes. When other factors that affect distance are taken into account, individuals tend to keep conspecifics of the same sex at a distance inversely proportional to their arm length, whereas they tend to keep conspecifics of a different sex at a distance inversely proportional to the degree of similarity between their body sizes: a man will let a woman with approximately equal long arms come closer than a smaller woman, and vice-versa.

While these results suggest that factors related to the body schema may account for effects that are widely reported as due to sex roles, they do not necessarily imply that all social and cultural factors are irrelevant to our understanding of personal distance. In the different-sex condition of the current study, for instance, along with a modulating effect of differences in arm length we did observe a (somewhat smaller) effect of participant sex: arm length differences being equal, male participants tended to let the female confederate come closer than did female participants with the male confederate. This difference is very likely to be related to different social norms and stereotypes regarding appropriate behaviors for male or females encountering 
members of the opposite sex.

In addition, the proxemics literature is rich in studies documenting the role of ethnicity in modulating proxemic distance. A recent study [30], for instance, measured spaces between 23 seated participants that were filling mock questionnaires. Results showed that Anglo Saxons sat at the largest distance, whereas Mediterraneans and Latinos preferrred shorter distances. This finding is consistent with earlier results [31-33]. Although we did avoid recruiting foreign students as participants, we did not control for participant's family region of origin. This is unlikely to explain our results as participants were assigned randomly to conditions. However, region of origin may account for part of our observed variability due to differences in cultural norms for personal space in, for instance, Southern and Northern Italians. It may also be noted, however, that at least some of the reported ethnicity effects may involve also a contribution from factors related to the body schema. Latinos and Mediterraneans also tend, on the average, to be somewhat shorter than Anglo Saxons or Scandinavians, as do Southern compared to Northern Italians.

\section{Conclusion}

The present results demonstrate that arm lenght modulates personal distance whereas sex plays only an indirect role in defining such distance. In same-sex pairs, in particular, the seeming difference between male and female pairs can be entirely explained by the effect of arm length in both men and women. Such an effect may reflect a role of action potentiality in modulating personal space: if you approach me, I keep you at a distance that is just-within-reach, but no closer. In different-sex pairs, the interpretation of the results is more complex. We propose that in this case the different effect of arm length in female-male vs. male-female pairs might reflect not only generic possibilities for action, but also the possibility of accepting or rejecting intimacy. If your body size resembles mine (such that our arms are similar in lenght), I am more likely to let you come closer. As an alternative, the pattern of results in the different-sex pairs could be interpreted as reflecting different social norms for males and females as they are approached by different-sex individuals. When men are approached by women, letting the woman come closer does not carry a negative connotation for the man. When female are approached by a strange man, conversely, there may be a tendency for the woman to keep the man farther away to avoid culturally negative implications. Within this alternative account, it is less clear however why these culturally-induced biases would produce opposite slopes as a function of arm length. Whatever the case, the results seem at odds with the prediction of standard proxemic theory.

The possibility that factors related one's body size play a role in setting the limits of the proxemic bubble of personal distance should be of interest to students of human communication in psychological, sociological, and anthropological contexts. It suggests that handbook accounts of the role of distance in human interactions need revision, at least in their current emphasis on the role of the sex of the interacting individuals. It also calls for reinvesigations of reported sociocultural factors. We suggest that, to these aims, the neuroscience of cognitive representations of the body [34] and of environmental constraints on different types of perceptual [35] and neuropsychological [36] space proves a source of useful insights and directions for clarifying how differences related to sex interact with body-related factors [37].

\section{Acknowledgements}

Based in part on Michela Muzzolini's master thesis at the University of Trieste. The authors thank Dr. Stefano Ghirlanda for useful comments on an earlier version of this manuscript. This work was supported in part from a start-up grant from the University of Parma.

\section{REFERENCES}

[1] E. T. Hall. The hidden dimension. New York: Doubleday, 1966.

[2] H. Hediger. Studies of the psychology and behaviour of captive animals in zoos and circuses. Butterworths Scientific Publications, 1955.

[3] J. von Uexküll. A Stroll Through the Worlds of Animals and Men: A Picture Book of Invisible Worlds. In C.H. Schiller (a cura di) Instinctive Behavior: The Development of a Modern Concept. New York: International Universities Press, Inc., 1957.

[4] M. Argyle. Bodily communication. New York: International Universities, 1975.

[5] J.K. Burgoon, J. L. Hale. Nonverbal expectancy violations: model elaboration and application to immediacy behavior. Communication monographs, 55, 58-79, 1988.

[6] M.A. Dosey, M. Meisels. Personal space and self-protection. Journal of Personality and Social Psychology, 11, 93-97, 1969.

[7] N. Felipe, R. Sommer. Invasions of personal space. Social Problems, 14, 206-214, 1966.

[8] R. Sommer.. Personal space: the behavioral basis of design. Prentice-Hall, Englewood Cliffs, 1969.

[9] R. D. Middlemist, E. S.Knowles, C.F. Matter. Personal space invasions in the lavatory: suggestive evidence for arousal. J Pers Soc Psychol, 33(5), 541-46, 1976.

[10] D. P. Kennedy, J. Glascher, J.M. Tyszka, R. Adolphs. Personal space regulation by the human amygdala. Nature Neuroscience, 12(10), 1226-1227, 2009.

[11] J.R. Aiello. Human Spatial Behavior. In D. Stokols and I. Altman (eds), Handbook of Environmental Psychology Vol 1, 
New York: Wiley, 1987.

[12] L.A. Hayduk. Personal space: An evaluative and orienting overview. Psychological Bulletin, 85, 117-134, 1978.

[13] L.A. Hayduk. Personal space: Where we now stand. Psychological Bulletin, 94, 293-335, 1983.

[14] L.A. Hayduk. The shape of personal space: an experimental investigation. Canadian Journal of Behavioral Science, 13, 274-87, 1981.

[15] G.W. Evans, R.B. Howard. Personal space. Psychological Bulletin, 60, 334-344, 1973.

[16] R. Gifford. Projected interpersonal distance and orientation choices: Personality, sex, and social situation. Social Psychology Quarterly, 45, 142-152, 1982.

[17] J.J. Hartnett, K.G. Bailey, F.W.J. Gibson. Personal space as influenced by sex and type of movement. Journal of Psychology, 76(2d Half), 139-44, 1970.

[18] D. Uzzell, N. Horne,. The influence of biological sex, sexuality and gender role on interpersonal distance. British Journal of Social Psychology 45(Pt 3), 579-97, 2006.

[19] J.A. DeVito. Human Communication. Boston: Allyn \& Bacon., 2002.

[20] M. Baldassare, S. Feller. Cultural variations in personal space: Theory, methods, and evidence. Ethos, 3, 481-503., 1975.

[21] E. Griffin, E. A first look at communication theory. New York: McGraw-Hill, 1991.

[22] G. Berlucchi, S.M. Aglioti. The body in the brain: neural bases of corporeal awareness. Trends in Neurosciences, 20(12), 560-64, 1997.

[23] M.R. Longo, P. Haggard. What is it like to have a body? Current Directions in Psychological Science, 21(2), 140-45, 2012.

[24] N. Holmes, C. Spence. Beyond the body schema: Visual, Prosthetic and Technological Contributions to Bodily Perception and Awareness. In G. Knoblich, I. M. Thornton, M. Grosjean \& M. Shiffrar, Human body perception from the inside out. New York, NY: Oxford University press, 14-64, 2006.

[25] P.W. Halligan G.R. Fink , J.C.Marshall , G.Vallar G. Spatial cognition: evidence from visual neglect. Trends Cogn Sci 7:125-133, 2003.

[26] G. Rizzolatti C.Scandolara, M. Matelli, M. Gentilucci. Afferent properties of periarcuate neurons in macaque monkeys. II. Visual properties. Behav Brain Res 2:147-163, 1981.

[27] M. R. Longo, S. F. Lourenco (2007). Space perception and body morphology: extent of near space scales with arm length. Exp Brain Res, 177(2), 285-90, 2007.

[28] L. Adams, D. Zuckerman (1991). The effect of lighting conditions on personal space requirements. The journal of general psychology, 118, 335-40, 1991.

[29] L.N. Ashton, M.E. Shaw, A. P. Worsham, Affective reactions to interpersonal distance by friends and strangers. Bulletin of the Psychonomic Society, 15: 306-308, 1980.

[30] C. M. J. Beaulieu. Intercultural study of personal space: A case study. Journal of Applied Social Psychology, 34, 794-805, 2004.

[31] C. Albas, D. Albas. Aligning actions: the case of subcultural proxemics. Canadian Ethnic Studies, 21, 74-82., 1989.

[32] D. Thompson, J. Baxter. Interpersonal distance spacing in two-person cross-cultural interactions. Man-Environment Systems, 3, 115-117, 1973.

[33] S. Li. How close is too close? A comparison of proxemic reactions of Singaporean Chinese to male intruders of four ethnicities. Perceptual and Motor Skills, 93(1), 124-26, 2001.

[34] H. De Preester, V. Knockaert. Body Image and Body Schema. Amsterdam: John Benjamins Publishing, 2005.

[35] J.E. Cutting, P.M. Vishton. Perceiving layout and knowing distances: The interaction, relative potency, and contextual use of different information about depth. In W. Epstein \& S. Rogers (Eds.) Perception of space and motion. (pp. 69-117). San Diego, CA: Academic Press, 1995.

[36] F. H. Previc. The neuropsychology of 3-D space. Psychological Bulletin, 124, 123-164, 1988.

[37] H. Stancey, M. Turner. Close women, distant men: line bisection reveals sex-dimorphic patterns of visuomotor performance in near and far space. British Journal of Psychology, 101(Pt 2), 293-309, 2010. 